\title{
Mono-everything: combined limits on dark matter production at colliders from multiple final states
}

\author{
Ning Zhou, ${ }^{1}$ David Berge ${ }^{2}$ and Daniel Whiteson ${ }^{1}$ \\ ${ }^{1}$ Department of Physics and Astronomy, University of California, Irvine, CA 92697 \\ ${ }^{2}$ GRAPPA Institute, University of Amsterdam, Netherlands
}

\begin{abstract}
Searches for dark matter production at particle colliders are complementary to direct-detection and indirect-detection experiments, and especially powerful for small masses, $m_{\chi}<100 \mathrm{GeV}$. An important collider dark matter signature is due to the production of a pair of these invisible particles with the initial-state radiation of a standard model particle. Currently, collider searches use individual and nearly orthogonal final states to search for initial-state jets, photons or massive gauge bosons. We combine these results across final states and across experiments to give the strongest current collider-based limits in the context of effective field theories, and map these to limits on dark matter interactions with nuclei and to dark matter self-annhiliation.
\end{abstract}

PACS numbers:

Though the presence of dark matter in the universe 22 has been well-established, little is known of its particle 23 nature or its non-gravitational interactions. A vibrant ${ }_{24}$ experimental program is searching for a weakly interact- 25 ing massive particle (WIMP), denoted as $\chi$, and inter- 26 actions with standard model particles via some as-yet- 27 unknown mediator. If the mediator is too heavy to be 28 resolved, the interaction can be modeled as an effective 29 field theory with a four-point interaction.

30

One critical component of this program is the search ${ }^{31}$ for pair-production of WIMPs at particle colliders, specif- 32 ically $p p \rightarrow \chi \bar{\chi}$ at the LHC via some unknown interme- ${ }^{33}$ diate state. As the final state WIMPs are invisible to ${ }^{34}$ the detectors, the events can only be seen if there is as- 35 sociated initial-state radiation of a standard model par- 36 ticle [1 3], see Fig 1, recoiling against the dark matter ${ }^{37}$ pair.

The LHC collaborations have reported limits on the ${ }^{39}$ cross section of $p p \rightarrow \chi \bar{\chi}+X$ where $X$ is a gluon or ${ }^{40}$ quark [4, 5], photon [6, 7, and other searches have been ${ }^{41}$ repurposed to study the cases where $X$ is a $W$ [ $]$ or $Z^{42}$

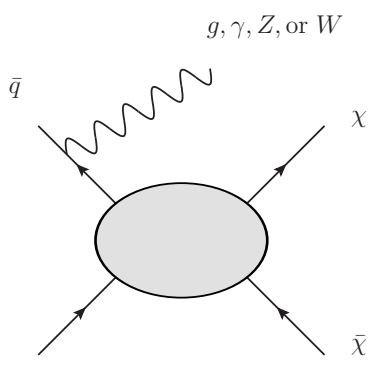

53

FIG. 1: Pair production of WIMPs $(\chi \bar{\chi})$ in proton-proton ${ }_{54}$ collisions at the LHC via an unknown intermediate state, with ${ }_{55}$ initial-state radiation of a standard model particle. boson [9, 10]. In each case, limits are reported in terms of the mass scale $M_{\star}$ of the unknown interaction expressed in an effective field theory [1-3, 12 20]. These various initial-state tags probe the same effective theory, but are largely statistically independent due to their nearly orthogonal event selection requirements. As the relative rates of radiation of gluons (quarks), photons, $W$ or $Z$ bosons from the incoming quark (gluon) legs are determined by the standard model, the various probes may be combined to give the strongest limits without any loss of generality or additional theoretical assumptions.

Recently, an analysis of multi-jet final states was shown to add some sensitivity to the mono-jet analyses 22 ; that sample is not statistically independent from the mono-jet results used here, and is not included. An earlier global analysis of indirect and direct constraints with Tevatron data and mono-jet data from ATLAS provided an initial set of combined constraints [23] using the approximations of a $\chi^{2}$ technique.

In this paper, we perform a full statistical combination of the limits from all available channels (mono-jet, mono-photon, mono- $Z^{1}$ from both ATLAS and CMS at $\sqrt{s}=7 \mathrm{TeV}$, accounting for the dominant correlations and providing the most powerful current collider constraints. While the limits reported by the experimental collaborations are typically given for a few select effective operators, we calculate the efficiencies of their selections and reinterpret their searches for the complete set of operators relevant for Dirac fermion or complex scalar WIMPs.

\section{Models}

The effective theories of dark matter considered here consider the possibility that the final-state WIMPs are a Dirac fermion (operators D1-D14 in Ref [15]) or a com-

\footnotetext{
${ }^{1}$ Final states with a heavy boson have little power relative to mono-photon or mono-jet; we include mono- $Z$ as a demonstration, and do not include mono- $W$, although see [8]. For an alternative view of mono- $Z$, see $\operatorname{Ref}[1]$
} 

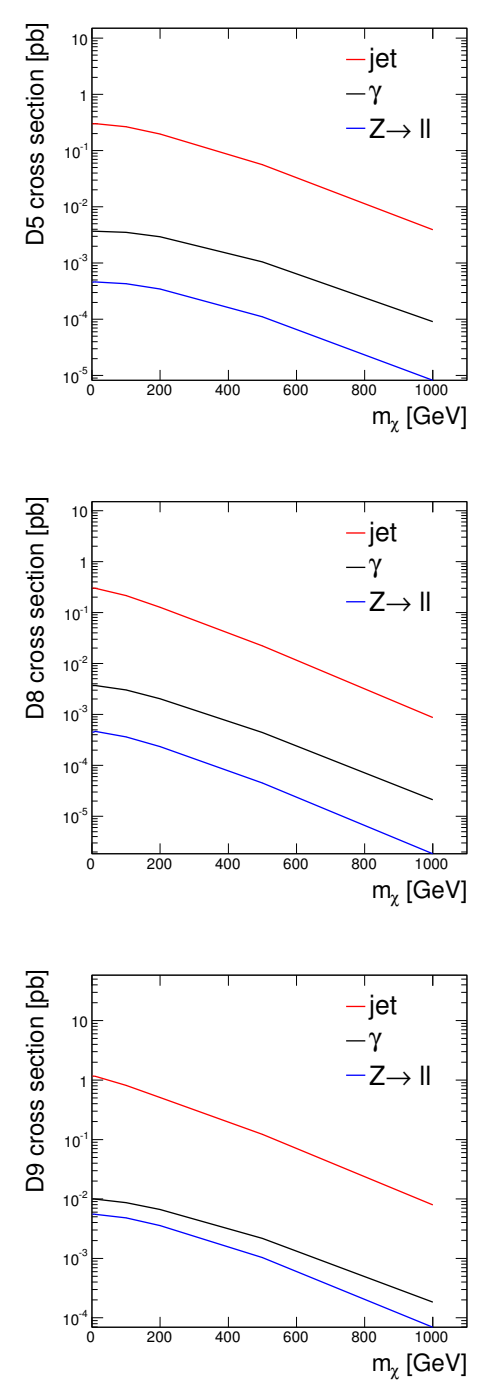

FIG. 2: Cross sections for $p p \rightarrow \chi \bar{\chi}+X$ production where $X$ is initial-state radiation of a jet, photon or $Z$ boson. Jet and ${ }^{97}$ photon final states include a $p_{T}>80 \mathrm{GeV}$ cut at parton-level. 98 Each pane shows the cross section for a different effective ${ }_{99}$ operator: top is D5, center is D8, bottom is D9. See Ref. 3] for operator definitions.

tions for WIMP-nucleon interaction, $\sigma(\chi-n)$ [3], or WIMP annihilations [2]. Therefore the effective field theories allow us to map measurements performed at the LHC to the quantities relevant for direct-detection and indirect-detection dark matter search experiments.

The effective-field-theory approach is valid as long as the unknown new mediator particles that couple the dark-matter particles to SM quarks or gluons are too heavy to be resolved: $q<M^{*}$, where $q$ is the momentum transfer. The breakdown of the effective approach depends ultimately on the details of the new and unknown physics, specifically on the number of new mediator particles and the new couplings. Therefore, these theories cannot be treated generically and must be interpretted with some care. To guide the interpretation, we indicate the range of validity as lower bounds on the mass suppression scale $M_{\star}$ following ref. 3. We note that any range of validity of the effective field theory involves assumptions about the unknown physics, see Refs 21 and 22 for additional unitarity arguments and more stringent validity ranges.

Assuming the simplest possible structure of new physics (mediation via exactly one new heavy mediator of mass $M, M_{\star}=M / \sqrt{g_{1} g_{2}}, g_{1}$ and $g_{2}$ being coupling constants), bounds on the suppression scale can be placed by requiring $M>2 m_{\chi}$ and that the new physics be as strongly coupled as possible for it to be still perturbative $\left(\sqrt{g_{1} g_{2}}<4 \pi\right)$ :

$$
\begin{aligned}
& M_{\star}>\frac{m_{\chi}}{2 \pi}(\mathrm{D} 5 \text { to } \mathrm{D} 14 \text { and } \mathrm{C} 3 \text { to } \mathrm{C} 6), \\
& \sqrt{\frac{M_{\star}^{3}}{m_{q}}}>\frac{m_{\chi}}{2 \pi} \text { (D1 to D4), } \\
& \frac{M_{\star}^{2}}{m_{q}}>\frac{m_{\chi}}{8 \pi^{2}}(\mathrm{C} 1 \text { and } \mathrm{C} 2) .
\end{aligned}
$$

Note that we are accounting for additional factors of $m_{q}$ in the definitions of operators D1 to D4 and C1, C2 of ref. [3].

\section{Experimental Searches}

The experimental searches typically require one or more high- $p_{\mathrm{T}}$ object and missing transverse momentum, see Table I for a summary and comparison of the monophoton and mono-jet selections.

The mono- $Z$ analysis [10] uses the ATLAS $Z Z \rightarrow \ell \ell \nu \nu$ cross-section measurement [9], which requires:

- two same-flavor opposite-sign electrons or muons, each with $p_{\mathrm{T}}^{\ell}>20 \mathrm{GeV},\left|\eta^{\ell}\right|<2.5$;

- dilepton invariant mass close to the $Z$ boson mass: $m_{\ell \ell} \in\left[m_{Z}-15, m_{Z}+15\right] \mathrm{GeV}$;

- no particle-level jet with $p_{\mathrm{T}}^{j}>25 \mathrm{GeV}$ and $\left|\eta^{j}\right|<4.5$
For some operators, cross sections of dark matter pro-111 duction at the LHC can be transformed into cross sec-112 
TABLE I: Summary of event selection requirements in ATLAS and CMS mono-jet or mono-photon analyses. Note that ATLAS uses two signal regions $\left(E_{T}>350\right.$ or $\left.500 \mathrm{GeV}\right)$ for the mono-jet analyses, depending on the operator.

\begin{tabular}{l|l|l}
\hline \hline & ATLAS & CMS \\
\hline jet & 1 or 2 jets & 1 or 2 jets \\
& $p_{\mathrm{T}}^{j_{1}}>350(500) \mathrm{GeV}$ & $p_{\mathrm{T}}^{j_{1}}>110 \mathrm{GeV}$ \\
& $p_{\mathrm{T}}^{j_{2}}>30 \mathrm{GeV}$ & $p_{\mathrm{T}}^{j_{2}}>30 \mathrm{GeV}$ \\
& $E_{T}>350(500) \mathrm{GeV}$ & $E_{T}>350 \mathrm{GeV}$ \\
& veto leptons & veto leptons \\
& $\Delta \phi\left(j_{2}, E_{T}\right)>0.5$ & $\Delta \phi\left(j_{1}, j_{2}\right)<2.5$ \\
\hline$\gamma$ & 1 photon, $p_{\mathrm{T}}>150 \mathrm{GeV}$ & 1 photon $p_{\mathrm{T}}>145 \mathrm{GeV}$ \\
& $E_{T}>150 \mathrm{GeV}$ & $E_{T}>130 \mathrm{GeV}$ \\
& $\leq 1$ jet with $p_{\mathrm{T}}>30 \mathrm{GeV}$ & 0 track with $p_{T}>20 \mathrm{GeV}$ \\
& isolation details & isolation details \\
& $\Delta \phi\left(\gamma, E_{T}\right)>0.4$ & \\
$\Delta \phi\left(j_{1}, E_{T}\right)>0.4$ & \\
veto leptons &
\end{tabular}

$$
\begin{aligned}
& \text { - }\left(\left|p_{\mathrm{T}}^{\nu \bar{\nu}}-p_{\mathrm{T}}^{Z}\right|\right) / p_{\mathrm{T}}^{Z}<0.6 \\
& \text { - }-p_{\mathrm{T}}^{\nu \bar{\nu}} \times \cos \left(\Delta \phi\left(p_{\mathrm{T}}^{\nu \bar{\nu}}, p_{\mathrm{T}}^{Z}\right)\right)>80 \mathrm{GeV} .
\end{aligned}
$$

The selection efficiency of each selection for each operator is given in Table $\mathrm{II}$ and were estimated in the following way. References [4] provide signal efficiency for several select operators; this efficiency is the product of geometric and kinematic acceptance of the selection criteria and object reconstruction efficiency. The object reconstruction efficiency depends on the details of the detector performance, but is largely independent of operator. The geometric and kinematic acceptances can be reliably estimated using parton-level simulated event samples. We measure the geometric and kinematic efficiency for each operator, and use the quoted total efficiences to deduce the object reconstruction efficiencies. This allows us to estimate the total efficiency for each operator.

\section{Combination}

The separate analyses, each of which are single-bin counting experiments, are combined into a multi-bin counting experiment. This allows for a coherent signal rate to be tested across channels, but preserves their distinct signal-to-background ratios.

The background estimates are taken directly from the experimental publications, see a summary in Table III. and are assumed to be uncorrelated across channels, as they are typically dominated by channel-specific or detector-specific uncertainties. For example, in some ${ }_{145}$ cases the background estimates are data-driven, and the ${ }_{146}$ dominant uncertainties are in the finite statistics of in-147 dependent control samples. Inclusion of correlations up 148 to $20 \%$ does not qualitatively impact the results of the 149 combination.
TABLE II: Selection efficiency as percentages for each channel of the analyses used in the combination, for operators D114 and C1-C6 for low and high values of the WIMP mass $m_{\chi}$. ATLAS mono-jet analysis has two signal regions, we use $E_{T}>500(350) \mathrm{GeV}$ and $p_{\mathrm{T}}^{j_{1}}>500(350) \mathrm{GeV}$ region for operators D9-D14 (D1-D8 and C1-C6). Operators D1114, C5 and C6 only couple to gluon initial states, and so have no efficiency for photon or $Z$ boson radiation. The $Z$ efficiencies include the $Z \rightarrow \ell \ell$ branching fraction. Jet and photon samples include a $p_{T}>80 \mathrm{GeV}$ cut at parton-level.

\begin{tabular}{lr|lrr|rr}
\hline \hline & & \multicolumn{3}{|c|}{ ATLAS } & \multicolumn{2}{|c}{ CMS } \\
Operator & $m_{\chi}$ & jet & $\gamma$ & $Z$ & jet & $\gamma$ \\
\hline D1 & 10 & $0.4 \%$ & $11.2 \%$ & $1.2 \%$ & $0.7 \%$ & $8.0 \%$ \\
& 1000 & $2.6 \%$ & $19.1 \%$ & $1.2 \%$ & $3.6 \%$ & $11.3 \%$ \\
\hline D2 & 10 & $0.4 \%$ & $10.8 \%$ & $1.2 \%$ & $0.7 \%$ & $8.0 \%$ \\
& 1000 & $2.4 \%$ & $18.6 \%$ & $1.1 \%$ & $3.7 \%$ & $11.3 \%$ \\
\hline D3 & 10 & $0.5 \%$ & $11.1 \%$ & $1.2 \%$ & $0.7 \%$ & $8.0 \%$ \\
& 1000 & $2.6 \%$ & $18.9 \%$ & $1.2 \%$ & $3.9 \%$ & $11.3 \%$ \\
\hline D4 & 10 & $0.5 \%$ & $10.8 \%$ & $1.2 \%$ & $0.7 \%$ & $7.6 \%$ \\
& 1000 & $2.6 \%$ & $18.6 \%$ & $1.1 \%$ & $3.7 \%$ & $11.3 \%$ \\
\hline D5 & 10 & $1.7 \%$ & $18.2 \%$ & $0.9 \%$ & $2.2 \%$ & $11.3 \%$ \\
& 1000 & $3.3 \%$ & $23.5 \%$ & $1.1 \%$ & $4.5 \%$ & $14.7 \%$ \\
\hline D6 & 10 & $1.7 \%$ & $18.7 \%$ & $0.9 \%$ & $2.2 \%$ & $12.0 \%$ \\
& 1000 & $3.2 \%$ & $23.6 \%$ & $1.1 \%$ & $4.4 \%$ & $15.2 \%$ \\
\hline D7 & 10 & $1.7 \%$ & $18.1 \%$ & $0.9 \%$ & $2.4 \%$ & $11.3 \%$ \\
& 1000 & $3.3 \%$ & $23.4 \%$ & $1.1 \%$ & $4.4 \%$ & $14.5 \%$ \\
\hline D8 & 10 & $1.7 \%$ & $18.5 \%$ & $0.9 \%$ & $2.3 \%$ & $11.8 \%$ \\
& 1000 & $3.1 \%$ & $23.6 \%$ & $1.1 \%$ & $4.3 \%$ & $15.1 \%$ \\
\hline D9 & 10 & $0.9 \%$ & $23.5 \%$ & $1.4 \%$ & $4.1 \%$ & $14.1 \%$ \\
& 1000 & $1.2 \%$ & $23.3 \%$ & $1.4 \%$ & $5.1 \%$ & $14.8 \%$ \\
\hline D10 & 10 & $1.1 \%$ & $23.6 \%$ & $1.4 \%$ & $4.2 \%$ & $14.4 \%$ \\
& 1000 & $1.2 \%$ & $23.4 \%$ & $1.4 \%$ & $5.2 \%$ & $14.8 \%$ \\
\hline D11 & 10 & $0.9 \%$ & - & - & $4.1 \%$ & - \\
& 1000 & $2.4 \%$ & - & - & $7.5 \%$ & - \\
\hline D12 & 10 & $1.0 \%$ & - & - & $4.2 \%$ & - \\
& 1000 & $2.4 \%$ & - & - & $7.4 \%$ & - \\
\hline D13 & 10 & $0.9 \%$ & - & - & $4.1 \%$ & - \\
& 1000 & $2.4 \%$ & - & - & $7.5 \%$ & - \\
\hline D14 & 10 & $1.1 \%$ & - & - & $4.0 \%$ & - \\
& 1000 & $2.4 \%$ & - & - & $7.4 \%$ & - \\
\hline C1 & 10 & $0.1 \%$ & $7.0 \%$ & $1.0 \%$ & $0.2 \%$ & $5.3 \%$ \\
& 1000 & $2.3 \%$ & $18.2 \%$ & $1.1 \%$ & $3.3 \%$ & $11.0 \%$ \\
\hline C2 & 10 & $0.1 \%$ & $7.0 \%$ & $1.0 \%$ & $0.1 \%$ & $5.6 \%$ \\
& 1000 & $2.5 \%$ & $18.4 \%$ & $1.1 \%$ & $3.8 \%$ & $11.2 \%$ \\
\hline C3 & 10 & $1.7 \%$ & $18.4 \%$ & $0.9 \%$ & $2.3 \%$ & $11.6 \%$ \\
& 1000 & $2.9 \%$ & $23.6 \%$ & $1.1 \%$ & $4.1 \%$ & $14.9 \%$ \\
\hline C4 & 10 & $1.4 \%$ & $18.4 \%$ & $0.9 \%$ & $2.2 \%$ & $11.8 \%$ \\
& 1000 & $3.0 \%$ & $23.8 \%$ & $1.1 \%$ & $4.1 \%$ & $15.3 \%$ \\
\hline C5 & 10 & $1.4 \%$ & - & - & $1.7 \%$ & - \\
& 1000 & $5.9 \%$ & - & - & $7.6 \%$ & \\
\hline C6 & 10 & $1.2 \%$ & - & - & $1.7 \%$ & - \\
& 1000 & $5.9 \%$ & - & $-7.6 \%$ & - \\
\hline \hline & & & & & & \\
& & & & &
\end{tabular}

The backgrounds, their uncertainties and the observed yield can be used to calculate a 90\% CL upper limit on the number of signal events $N$ in the sample, see Table III and Table IV] using the CLs method 25, 26. This value is almost completely model independent. Translating it into a limit on the cross section for the $p p \rightarrow \chi \bar{\chi}+X$ sig- 
TABLE III: $90 \%$ CL limits on $N_{\text {events }}$, efficiencies for $m_{\chi}=10$ $\mathrm{GeV}$, and limits on $\sigma(p p \rightarrow \chi \bar{\chi}+X)$ using the D5 operator. In the case of the $Z+E_{T}$ final state, the efficiency is relative to $Z \rightarrow \ell \ell$ decays only.

\begin{tabular}{lllrrrr}
\hline \hline Channel & Bg. & Obs & $\begin{array}{r}\text { Limit } \\
N\end{array}$ & $\begin{array}{r}\text { Eff. Lumi. } \\
\left(\mathrm{fb}^{-1}\right)\end{array}$ & $\begin{array}{r}\text { Limit } \\
(\mathrm{fb})\end{array}$ \\
\hline ATLAS jet+ $E_{T}$ & $750 \pm 60$ & 785 & 139.3 & $1.7 \%$ & 4.8 & 1,700 \\
CMS jet $+E_{T}$ & $1225 \pm 101$ & 1142 & 125.2 & $2.2 \%$ & 5.0 & 1,140 \\
ATLAS $\gamma+E_{T}$ & $137 \pm 20$ & 116 & 27.4 & $18 \%$ & 4.6 & 33 \\
CMS $\gamma+E_{T}$ & $75.1 \pm 9.4$ & 73 & 19.3 & $11 \%$ & 5.0 & 35 \\
ATLAS $Z+E_{T}$ & $86.2 \pm 7.2$ & 87 & 21.7 & $13 \%$ & 4.6 & 36 \\
\hline \hline
\end{tabular}

TABLE IV: $90 \%$ CL limits on $N_{\text {events, }}$ efficiencies for $m_{\chi}=10_{185}$ $\mathrm{GeV}$, and limits on $\sigma(p p \rightarrow \chi \bar{\chi}+X)$ using the D9 operator. ${ }_{186}$

\begin{tabular}{|c|c|c|c|c|c|c|}
\hline Channel & Bg. & Obs & $\begin{array}{r}\text { Limit } \\
N\end{array}$ & Eff. & $\begin{array}{l}\text { Lumi. } \\
\left(\mathrm{fb}^{-1}\right)\end{array}$ & $\begin{array}{c}\text { Limit } \\
\sigma(\mathrm{fb})\end{array}$ \\
\hline ATLAS jet+ $\mathscr{E}_{T}$ & $83 \pm 14$ & 77 & 25.5 & $0.9 \%$ & 4.8 & 590 \\
\hline $\mathrm{CMS}$ jet $+E_{T}$ & $1225 \pm 101$ & 1142 & 125.2 & $4.1 \%$ & 5.0 & 610 \\
\hline
\end{tabular}

nal requires the effieciency of the signal in each selection, ${ }^{192}$ see Table III These individual limits reproduce well the ${ }^{193}$ results reported by the experiments.

The signal regions are nearly orthogonal, but not $\mathrm{ex}^{-{ }^{195}}$ actly. For example, the mono-jet analyses do not veto ${ }^{190}$ events with a photon, and the mono-photon analyses al- ${ }^{197}$ low the presence of one jet. From our parton-level sim- ${ }^{198}$ ulated event samples, we estimated the overlaps among different channels and found that the overlap fraction is ${ }^{200}$ less than $1 \%$.

The individual analyses include signal uncertainties of up to $20 \%$ on the cross section, mostly due to uncertain- ${ }^{20}$ ties in jet energy calibration and levels of initial-state radiation. These uncertainties do not affect the cross $^{-202}$ section limits, but can be simply applied to limits on $^{203}$ $M_{\star}$. In each case, we quote the limit using the central ${ }^{204}$ value.

To summarize, the assumptions made in this combina ${ }^{200}$ tion are

- the background uncertainties are monolithic $\operatorname{and}_{209}$ uncorrelated, and

- the signal selections are orthogonal

Combining channels is then straightforward, though the intermediate step of a model-independent limit on ${ }^{212}$ the number of events $N$ is no longer possible, as the limits depend on the relative distribution of signal event s213 $_{21}$ across channels, which is model specific. Instead, cross-214 section limits are obtained directly. These limits are 215 then converted into limits on $M_{\star}$, using the relationships216 from Ref. 15. The individual-channel limits, combina-217 tion across experiments and the grand combination of all 218 channels are shown in Table $\mathrm{V}$ for the D5 operator and ${ }_{219}$ one choice of $m_{\chi}$. Clearly the mono-jet analyses are thez20 most powerful, and the greatest gain in combination is221
TABLE V: $90 \%$ CL limits on $\sigma(p p \rightarrow \chi \bar{\chi}+X)$ for $m_{\chi}=10$ $\mathrm{GeV}$, theory prediction for $M_{\star}=1 \mathrm{TeV}$, and limits on $M_{\star}$ using the D5 operator. In the case of the $Z+E_{T}$ final state, the prections include the $Z \rightarrow \ell \ell$ branching fraction.

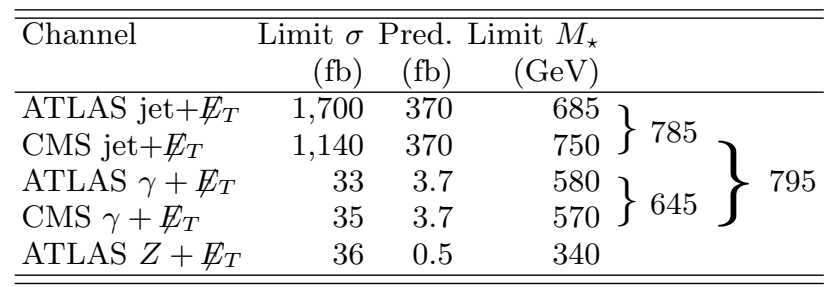

from combining the ATLAS and CMS mono-jet analyses, though the addition of the mono-photon and mono$Z$ gives a non-negligible improvement in the combined result.

Limits on $M_{\star}$ for the D5 and D8 operators are shown in Fig 3 and 4 , as well as limits on $\sigma(\chi-n)$. Where the $M_{\star}$ limits exceed the thermal relic values taken from Ref. 3], assuming that dark matter is entirely composed of thermal relics, the resulting dark matter density of the universe would contradict WMAP measurements; therefore, WIMPs cannot couple to quarks or gluons exclusively via the given operator and account entirely for the relic density. This $m_{\chi}$ region is either excluded, or requires that annihilation channels to leptons must exist, or participation of different operators which interfere negatively, thereby reducing the limits on $M_{\star}$.

\section{Application to other models}

While the experimental results are usually quoted for a small selection of the effective operator models, the analyses are clearly relevant for all of them.

We re-interpret the experimental analyses in the context of each operator and perform the grand combination across all channels. Figure 5 and Table VI show the limits on $M_{\star}$, translated to the WIMP-nucleon cross section where possible. In addition, we translate the limits on D5 and D8 into limits on the WIMP annihilation cross section, see Fig. 6 .

\section{Conclusions}

We have presented the first combination of colliderbased searches for dark matter pair production, using final states involving jets, photons and leptonicallydecaying $Z$ bosons in the context of effective field theories. The most powerful results are from the mono-jet analyses, and the greatest gains come from the combination of the independent analyses from ATLAS and CMS, though the other final states make a non-negligble improvement. The results are the strongest limits to date 

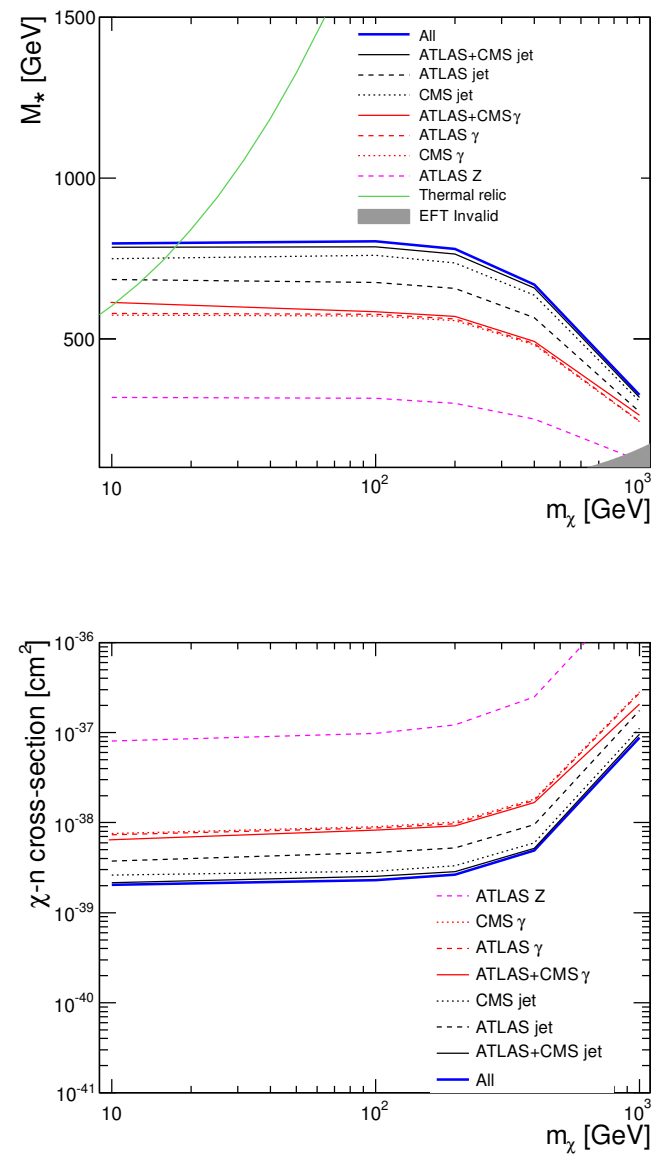

FIG. 3: Limits at 90\% CL in $M_{\star}$ (top) and in the spinindependent WIMP-nucleon cross section (bottom) for individual and combined limits using the D5 operator as a function of $m_{\chi}$.

from collider searches in the effective field theory context. ${ }^{237}$

In addition, we have reinterpreted the experimental re-20 sults, quoted by ATLAS and CMS only for a few effective operators, across a broad range of operators, providing a comprehensive view of the power of these searches to constrain the weak-level or weaker interactions between dark matter and standard model particles.

We have made use of the effective field theory frame-244 work to convert the ATLAS and CMS results to quan-245 tities relevant for direct-detection and indirect-detection ${ }^{246}$ dark matter searches. Under the assumptions made for $_{248}^{247}$ the effective operators, LHC limits can be very compet- ${ }^{248}$ itive, in particular for low-mass dark matter particles ${ }_{250}$ $m_{\chi} \leq 10 \mathrm{GeV}$.
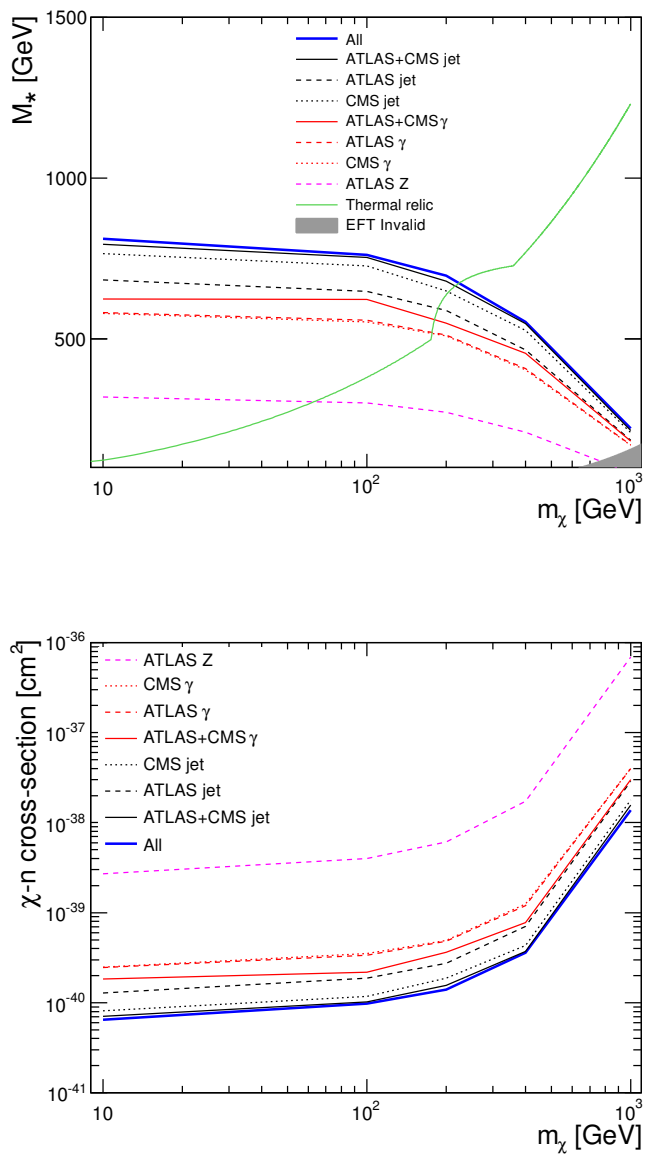

FIG. 4: Limits at $90 \% \mathrm{CL}$ in $M_{\star}$ (top) and in the spindependent WIMP-nucleon cross section (bottom) for individual and combined limits using the D8 operator as a function of $m_{\chi}$.

\section{Acknowledgements}

We acknowledge useful conversations with Tim Tait, Roni Harnik and Patrick Fox. DW and NZ are supported by grants from the Department of Energy Office of Science and by the Alfred P. Sloan Foundation.

[1] M. Beltran, D. Hooper, E. W. Kolb, Z. A. C. Krusberg and T. M. P. Tait, JHEP 1009, 037 (2010) arXiv:1002.4137 [hep-ph]].

[2] P. J. Fox, R. Harnik, J. Kopp and Y. Tsai, Phys. Rev. D 85, 056011 (2012) arXiv:1109.4398 [hep-ph]].

[3] J. Goodman, M. Ibe, A. Rajaraman, W. Shepherd, T. M. P. Tait and H. -B. Yu, Phys. Rev. D 82, 116010 (2010) arXiv:1008.1783 [hep-ph]].

[4] G. Aad et al. [ATLAS Collaboration], arXiv:1210.4491 [hep-ex].

[5] S. Chatrchyan et al. [CMS Collaboration], JHEP 1209, 

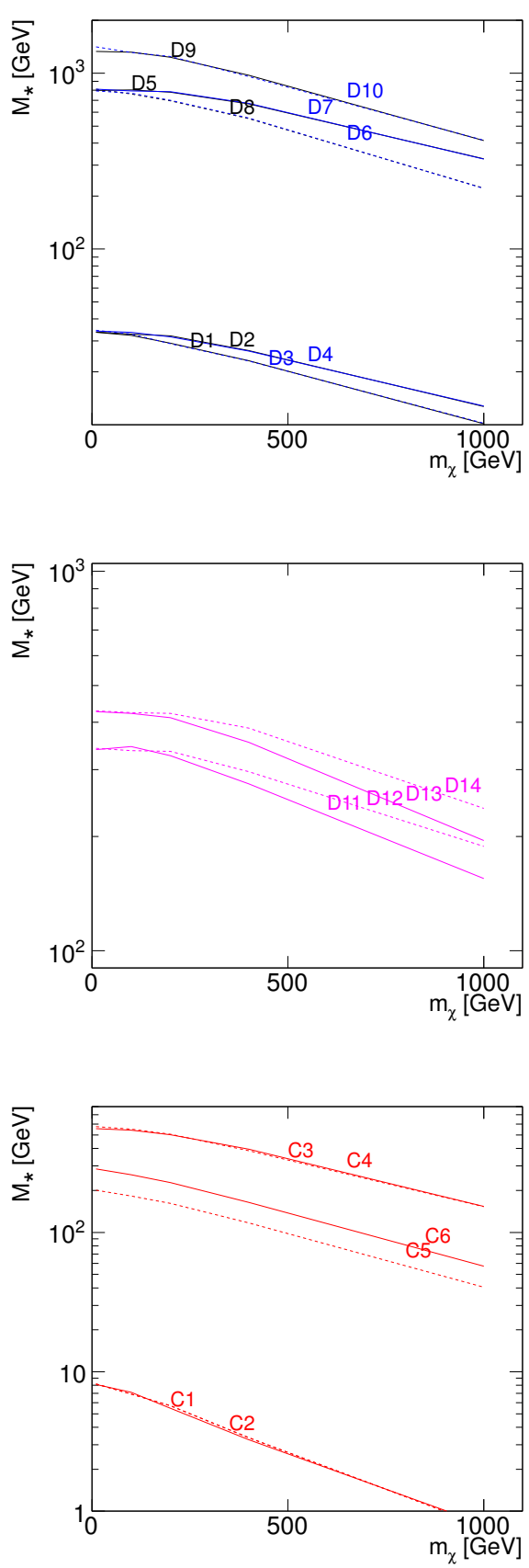

FIG. 5: Combined limits on $M_{\star}$ at $90 \%$ CL, using all available channels, for operators D1-14 and C1-C5 as a function of $m_{\chi}$.
094 (2012) arXiv:1206.5663 [hep-ex]].

[6] G. Aad et al. [ATLAS Collaboration], arXiv:1209.4625:62 [hep-ex].

[7] S. Chatrchyan et al. [CMS Collaboration], Phys. Rev.264 Lett. 108, 261803 (2012) arXiv:1204.0821 [hep-ex]]. ${ }_{265}$

[8] Y. Bai and T. M. P. Tait, arXiv:1208.4361 [hep-ph]. $\quad 260$

[9] G. Aad et al. [ATLAS Collaboration], arXiv:1211.6096 [hep-ex].

[10] L. M. Carpenter, A. Nelson, C. Shimmin, T. M. P. Tait269
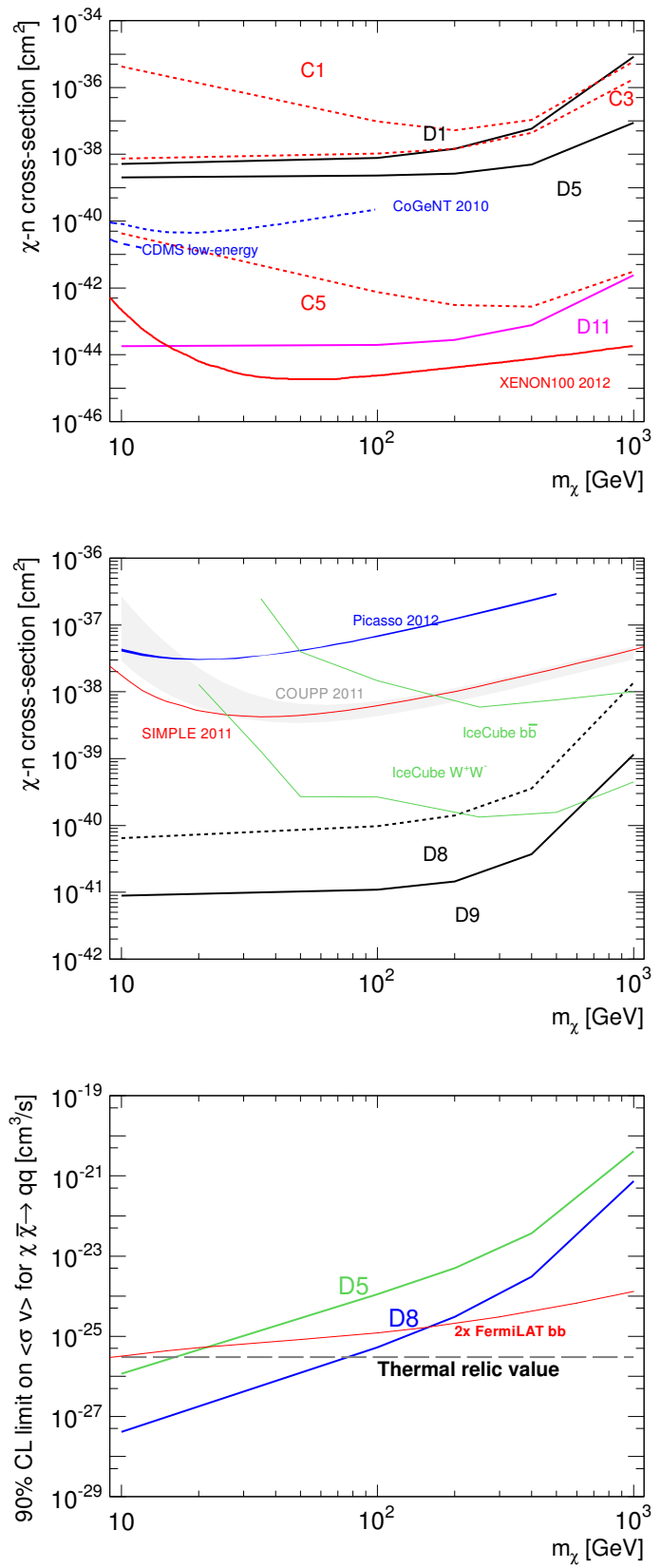

FIG. 6: Top and center, limits at 90\% CL on the spinindependent and spin-dependent WIMP-nucleon cross section, $\sigma(\chi-n)$, for available operators. Bottom, interpretation of the limits on D5 and D8 in terms of the velocity-averaged WIMP-annhiliation cross section, as defined in Ref [2].

and D. Whiteson, arXiv:1212.3352 [hep-ex].

[11] N. F. Bell, J. B. Dent, A. J. Galea, T. D. Jacques, L. M. Krauss and T. J. Weiler, Phys. Rev. D 86, 096011 (2012) [arXiv:1209.0231 [hep-ph]].

[12] M. Beltran, D. Hooper, E. W. Kolb and Z. C. Krusberg, Phys. Rev. D 80, 043509 (2009) arXiv:0808.3384 [hep$\mathrm{ph}]$.

[13] W. Shepherd, T. M. P. Tait and G. Zaharijas, Phys. Rev. D 79, 055022 (2009) arXiv:0901.2125 [hep-ph]]. 
TABLE VI: Combined limits on $M_{\star}$ at $90 \% \mathrm{CL}$, using all ${ }_{284}^{283}$ available channels, for operators D1-11 and C1-C5 for low and ${ }_{285}^{284}$ high values of the WIMP mass $m_{\chi}$. Where possible, limits are shown on the WIMP-nucleon cross section, $\sigma(\chi-n)$.

\begin{tabular}{|c|c|c|}
\hline Operator & $\begin{array}{r}m_{\chi} \\
(\mathrm{GeV})\end{array}$ & $\begin{array}{rr}M_{\star} & \sigma(\chi-n) \\
(\mathrm{GeV}) & {\left[\mathrm{cm}^{2}\right]}\end{array}$ \\
\hline \multirow[t]{2}{*}{ D1 } & 10 & $345.2 \times 10^{-39}$ \\
\hline & 1000 & $108.3 \times 10^{-36}$ \\
\hline \multirow[t]{2}{*}{ D2 } & 10 & 34 \\
\hline & 1000 & 13 \\
\hline \multirow[t]{2}{*}{ D3 } & 10 & 34 \\
\hline & 1000 & 10 \\
\hline \multirow[t]{2}{*}{$\mathrm{D} 4$} & 10 & 34 \\
\hline & 1000 & 13 \\
\hline \multirow[t]{2}{*}{ D5 } & 10 & $7952.0 \times 10^{-39}$ \\
\hline & 1000 & $3258.8 \times 10^{-38}$ \\
\hline \multirow[t]{2}{*}{ D6 } & 10 & 791 \\
\hline & 1000 & 221 \\
\hline \multirow[t]{2}{*}{ D7 } & 10 & 812 \\
\hline & 1000 & 324 \\
\hline \multirow[t]{2}{*}{ D8 } & 10 & $8116.5 \times 10^{-41}$ \\
\hline & 1000 & $2221.4 \times 10^{-38}$ \\
\hline \multirow[t]{2}{*}{ D9 } & 10 & $13318.9 \times 10^{-42}$ \\
\hline & 1000 & $4131.1 \times 10^{-39}$ \\
\hline \multirow[t]{2}{*}{ D10 } & 10 & 1410 \\
\hline & 1000 & 415 \\
\hline \multirow[t]{2}{*}{ D11 } & 10 & $3391.8 \times 10^{-44}$ \\
\hline & 1000 & $1552.4 \times 10^{-42}$ \\
\hline \multirow[t]{2}{*}{ D12 } & 10 & 342 \\
\hline & 1000 & 188 \\
\hline \multirow[t]{2}{*}{ D13 } & 10 & 427 \\
\hline & 1000 & 195 \\
\hline \multirow[t]{2}{*}{ D14 } & 10 & 429 \\
\hline & 1000 & 237 \\
\hline \multirow[t]{2}{*}{$\mathrm{C} 1$} & 10 & $84.2 \times 10^{-36}$ \\
\hline & 1000 & $16.1 \times 10^{-36}$ \\
\hline \multirow[t]{2}{*}{$\mathrm{C} 2$} & 10 & 8 \\
\hline & 1000 & 1 \\
\hline \multirow[t]{2}{*}{ C3 } & 10 & $5757.5 \times 10^{-39}$ \\
\hline & 1000 & $1531.8 \times 10^{-36}$ \\
\hline \multirow[t]{2}{*}{$\mathrm{C} 4$} & 10 & 556 \\
\hline & 1000 & 154 \\
\hline \multirow[t]{2}{*}{$\mathrm{C} 5$} & 10 & $2014.4 \times 10^{-41}$ \\
\hline & 1000 & $413.1 \times 10^{-42}$ \\
\hline \multirow[t]{2}{*}{ C6 } & 10 & 286 \\
\hline & 1000 & 57 \\
\hline
\end{tabular}

[14] Q. -H. Cao, C. -R. Chen, C. S. Li and H. Zhang, JHEP 1108, 018 (2011) arXiv:0912.4511 [hep-ph]].

[15] J. Goodman, M. Ibe, A. Rajaraman, W. Shepherd, T. M. P. Tait and H. -B. Yu, Phys. Lett. B 695, 185 (2011) arXiv:1005.1286 [hep-ph]].

[16] Y. Bai, P. J. Fox and R. Harnik, JHEP 1012, 048 (2010) arXiv:1005.3797 [hep-ph]].

[17] A. Rajaraman, W. Shepherd, T. M. P. Tait and A. M. Wijangco, Phys. Rev. D 84, 095013 (2011) arXiv:1108.1196 [hep-ph]].

[18] R. C. Cotta, J. L. Hewett, M. P. Le and T. G. Rizzo, arXiv:1210.0525 [hep-ph].

[19] F. J. Petriello, S. Quackenbush and K. M. Zurek, Phys.
Rev. D 77, 115020 (2008) arXiv:0803.4005 [hep-ph]].

[20] Y. Gershtein, F. Petriello, S. Quackenbush and K. M. Zurek, Phys. Rev. D 78, 095002 (2008) arXiv:0809.2849 [hep-ph]].

[21] I. M. Shoemaker, L. Vecchi and, Phys. Rev. D 86, 015023 (2012) arXiv:1112.5457 [hep-ph]].

[22] P. J. Fox, R. Harnik, R. Primulando and C. -T. Yu, Phys. Rev. D 86, 015010 (2012) arXiv:1203.1662 [hep-ph]].

[23] K. Cheung, P. -Y. Tseng, Y. -L. S. Tsai and T. -C. Yuan, JCAP 1205, 001 (2012) arXiv:1201.3402 [hep-ph]].

[24] P. J. Fox and C. Williams, arXiv:1211.6390 [hep-ph].

[25] A. Read, J. Phys. G: Nucl. Part. Phys. 28, 2693 (2002);

[26] T. Junk, Nucl. Instrum. Methods A 434, 425 (1999).

[27] J. Alwall, M. Herquet, F. Maltoni, O. Mattelaer and T. Stelzer, JHEP 1106, 128 (2011) arXiv:1106.0522 [hep-ph]]. 


\section{Appendix: Individual Operators}

In Figs. 7, 8, 9, 10 11, and 12 we show the combined 307 limits for each operator, compared to the thermal relic ${ }^{308}$ values. Where the limits exceed the thermal relic val-309 ues, assuming that dark matter is entirely composed of 310 thermal relics, the dark matter density of the universe This $m_{\chi}$ region is either excluded, or else other annihilation channels to leptons must exist, or finally different operators may interfere negatively thereby reducing the limits on $M_{\star}$. 

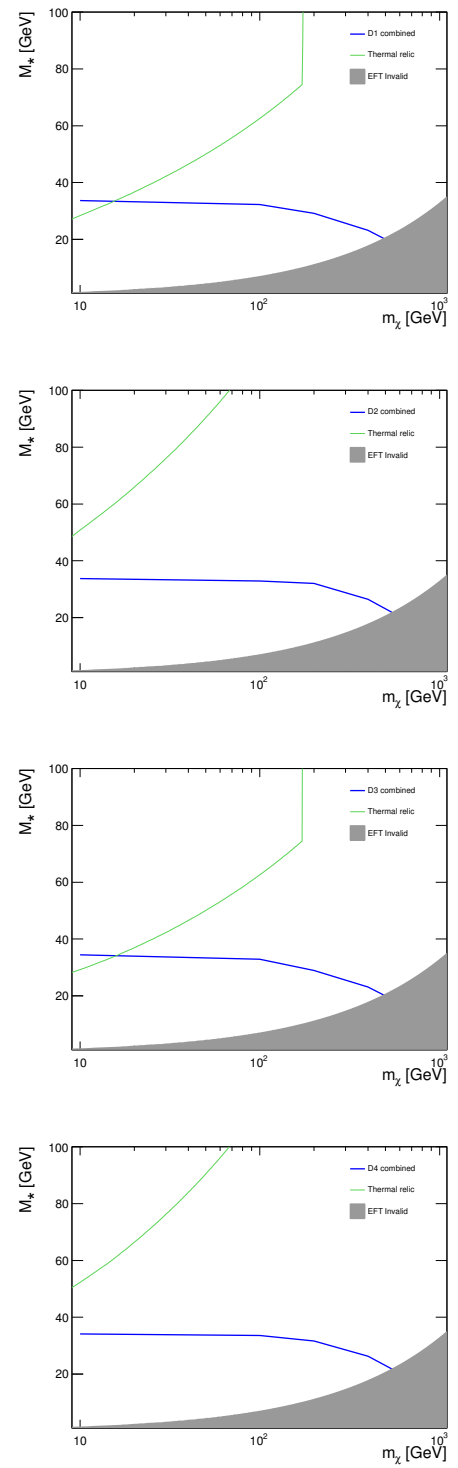

FIG. 7: Combined limits on $M_{\star}$ versus dark matter mass $m_{\chi}$ for operators D1, D2, D3 and D4. The $M_{\star}$ values at which dark matter particles of a given mass would result in the required relic abundance are shown as green lines [3], assuming annihilation in the early universe proceeded exclusively via the given operator. 

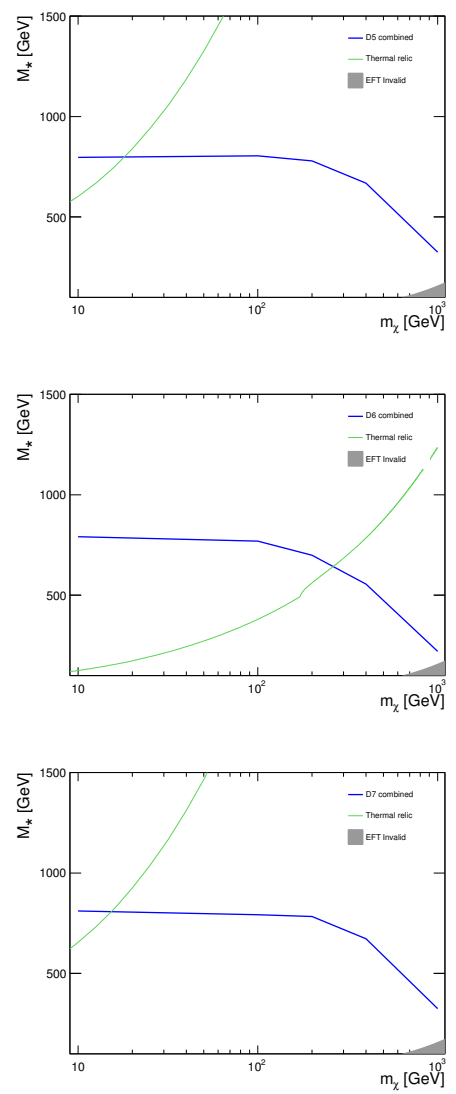

FIG. 8: Combined limits on $M_{\star}$ versus dark matter mass $m_{\chi}$ for operators D5, D6, and D7. The $M_{\star}$ values at which dark matter particles of a given mass would result in the required relic abundance are shown as green lines [3], assuming annihilation in the early universe proceeded exclusively via the given operator. 

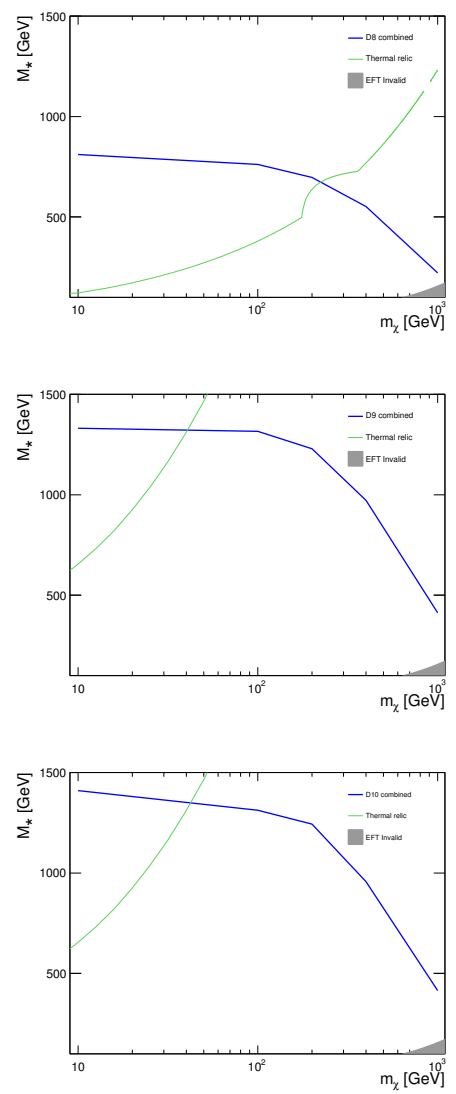

FIG. 9: Combined limits on $M_{\star}$ versus dark matter mass $m_{\chi}$ for operators D8,D9 and D10. The $M_{\star}$ values at which dark matter particles of a given mass would result in the required relic abundance are shown as green lines [3], assuming annihilation in the early universe proceeded exclusively via the given operator. 

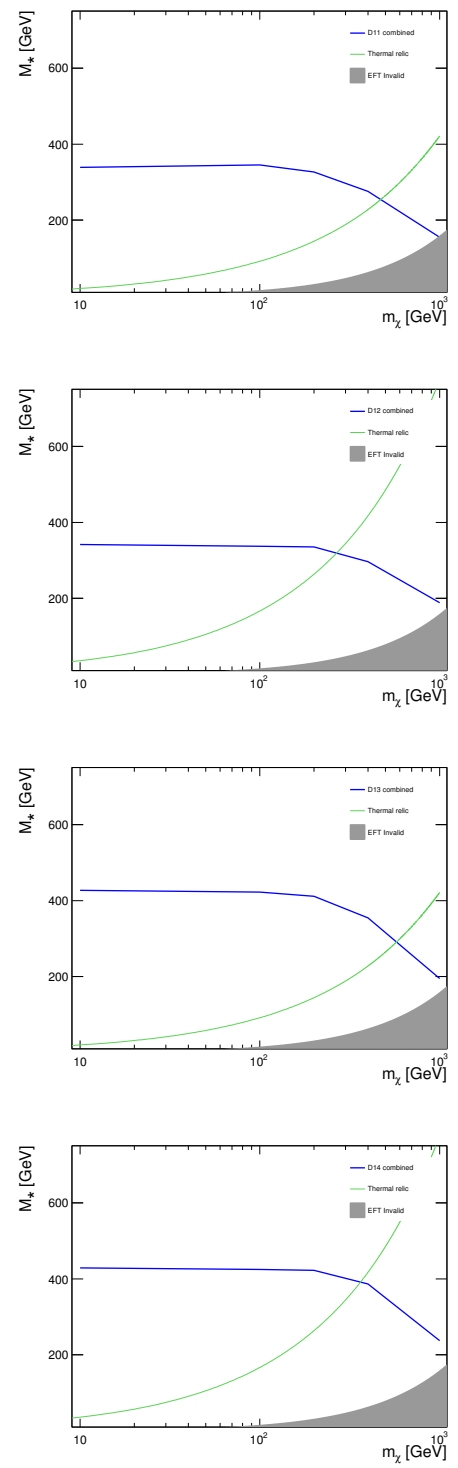

FIG. 10: Combined limits on $M_{\star}$ versus dark matter mass $m_{\chi}$ for operators D11, D12, D13 and D14. The $M_{\star}$ values at which dark matter particles of a given mass would result in the required relic abundance are shown as green lines [3], assuming annihilation in the early universe proceeded exclusively via the given operator. 

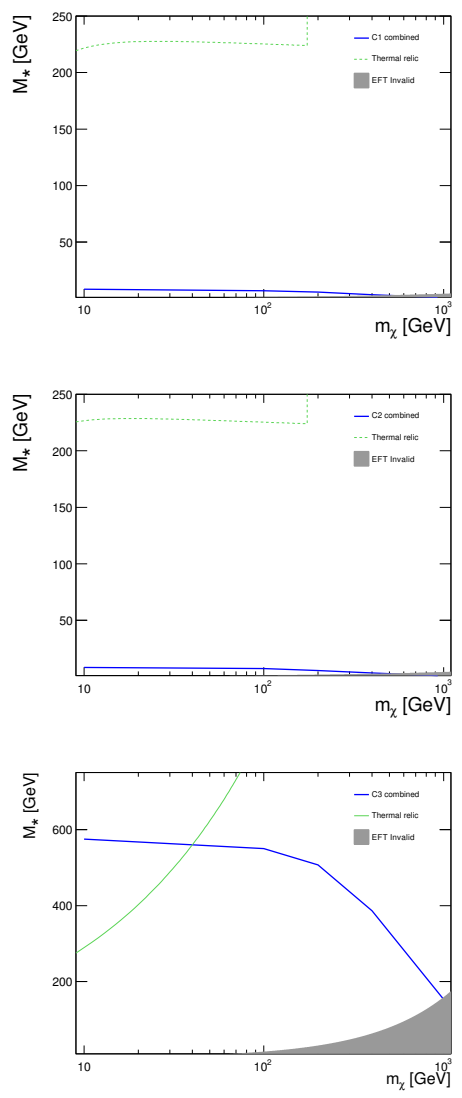

FIG. 11: Combined limits on $M_{\star}$ versus dark matter mass $m_{\chi}$ for operators $\mathrm{C} 1, \mathrm{C} 2$, and C3. The $M_{\star}$ values at which dark matter particles of a given mass would result in the required relic abundance are shown as green lines [3], assuming annihilation in the early universe proceeded exclusively via the given operator. 

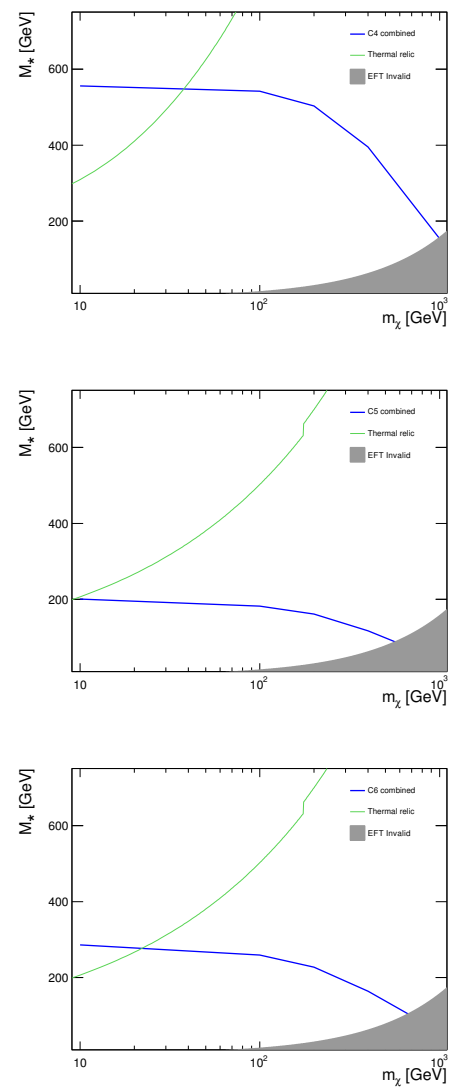

FIG. 12: Combined limits on $M_{\star}$ versus dark matter mass $m_{\chi}$ for operators $\mathrm{C} 4, \mathrm{C} 5$ and C6. The $M_{\star}$ values at which dark matter particles of a given mass would result in the required relic abundance are shown as green lines [3], assuming annihilation in the early universe proceeded exclusively via the given operator. 